\title{
What constitutes urgent endoscopy? A social media snapshot of gastroenterologists' views during the COVID-19 pandemic
}

\section{다(1)이우}

\author{
Authors \\ Mohammad Bilal' ${ }^{1}$, Malorie Simons², Asad Ur Rahman³ ${ }^{3}$ Zachary L. Smith ${ }^{4}$, Shifa Umar ${ }^{5}$, Jonah Cohen ${ }^{1}$, Mandeep S. \\ Sawhney ${ }^{1}$, Tyler M. Berzin'1, Douglas K. Pleskow ${ }^{1}$
}

Institutions

1 Center for Advanced Endoscopy, Division of Gastroenterology, Beth Israel Deaconess Medical Center, Harvard Medical School, Boston, Massachusetts, United States

2 Division of Gastroenterology \& Hepatology, Johns Hopkins Hospital, Baltimore, Maryland, United States

3 Department of Gastroenterology \& Hepatology, Cleveland Clinic Florida, Weston, Florida, United States

4 Division of Gastroenterology and Liver Disease, University Hospitals \& Case Western Reserve University School of Medicine, Cleveland, Ohio, United States

5 Division of Gastroenterology \& Hepatology, Allegheny Health Network, Pittsburgh, Pennsylvania, United States

submitted 2.4 .2020

accepted after revision 3.4 .2020

Bibliography

DOI https://doi.org/10.1055/a-1153-9014 |

Endoscopy International Open 2020; 08: E693-E698

(c) Georg Thieme Verlag KG Stuttgart · New York

elSSN 2196-9736

Corresponding author

Mohammad Bilal, MD, Center for Advanced Endoscopy, Division of Gastroenterology, Beth Israel Deaconess Medical Center, Harvard Medical School, 330 Brookline Ave, Boston, MA 02115

Fax: +1-617-667-8098

mbilal1@bidmc.harvard.edu

\section{ABSTRACT}

Background and study aims There is a consensus among gastroenterology organizations that elective endoscopic procedures should be deferred during the COVID-19 pan- demic. While the decision to perform urgent procedures and to defer entirely elective procedures is mostly evident, there is a wide "middle ground" of time-sensitive but not technically urgent or emergent endoscopic interventions. We aimed to survey gastroenterologists worldwide using Twitter to help elucidate these definitions using commonly encountered clinical scenarios during the COVID-19 pandemic.

Methods A 16-question survey was designed by the authors to include common clinical scenarios that do not have clear guidelines regarding the timing or urgency of endoscopic evaluation. This survey was posted on Twitter. The survey remained open to polling for 48 hours. During this time, multiple gastroenterologists and fellows with prominent social media presence were tagged to disseminate the survey.

Results The initial tweet had 38,795 impressions with a total of 2855 engagements. There was significant variation in responses from gastroenterologists regarding timing of endoscopy in these semi-urgent scenarios. There were only three of 16 scenarios for which more than $70 \%$ of gastroenterologists agreed on procedure-timing. For example, significant variation was noted in regard to timing of upper endoscopy in patients with melena, with $44.5 \%$ of respondents believing that everyone with melena should undergo endoscopic evaluation at this time. Similarly, about $35 \%$ of respondents thought that endoscopic retrograde cholangiopancreatography should only be performed in patients with choledocholithiasis with abdominal pain or jaundice. Conclusion Our analysis shows that there is currently lack of consensus among gastroenterologists in regards to timing of semi-urgent or non-life-threatening procedures during the COVID-19 pandemic. These results support the need for the ongoing development of societal guidance for these "semi-urgent" scenarios to help gastroenterologists in making difficult triage decisions. 


\section{Introduction}

In March 2020, the World Health Organization (WHO) declared the SARS-CoV-2/Novel Coronavirus-19 (COVID-19) a global pandemic. As of March 23, 2020, more than 300,000 cases have been reported worldwide [1]. Patients can present with varying degrees of symptoms and according to one report, $86 \%$ of all infections were undocumented prior to January 23 , 2020 [2]. Therefore, risk of infection reported in healthcare workers is substantial [3]. In particular, while performing gastrointestinal endoscopy, there is risk of exposure to the endoscopists as well as the endoscopy team, including nurses, endoscopy technicians and anesthesia staff [4]. While upper gastrointestinal endoscopy is an aerosol-generating procedure, there are now data to suggest that the risk may not limited to upper endoscopy alone, as recent reports have detected SARS-CoV in stool samples [5]. This has led to recommendations that all elective and non-urgent endoscopic procedures be cancelled or postponed at this time [4,6].

However, important questions have emerged regarding how to define an urgent procedure vs a non-urgent procedure, or a procedure that can be deferred for a discrete period of time. In some clinical scenarios, the decision to perform or delay a procedure is evident. For example, there is clear consensus that procedures for indications such as suspected variceal bleeding, non-variceal upper gastrointestinal bleeding, acute cholangitis, foreign body removal, and cancer-related care (i.e. tissue acquisition for diagnosis, loco-regional staging, and palliative procedures) are urgent and should continue to be performed [7-9]. Similarly, endoscopic evaluations of chronic symptoms such as diarrhea and gastroesophageal reflux disease (GERD), or screening for colorectal cancer in average-risk individuals, are considered non-urgent and should be deferred. Between these definitions exists a large array of potentially time-sensitive but not technically urgent or emergent endoscopic interventions. These grey areas or "semi-urgent" indications pose a clinical dilemma for the gastroenterologist with regard to proceeding with or deferring the procedure during this unprecedented time.

We aimed to survey gastroenterologists worldwide using Twitter to help elucidate these definitions using commonly encountered clinical scenarios during the COVID-19 pandemic. We hypothesized that there would be significant variability regarding procedures and indications considered urgent or nonurgent, highlighting the need for further guidance and standardization in identifying time-sensitive procedures.

\section{Methods}

A 12-question survey was designed by the authors. The goal was to choose common clinical scenarios that do not have clear guidelines regarding the timing or urgency of endoscopic evaluation or treatment during the current COVID-19 pandemic [4, $6]$. These questions were posted on Twitter using the "Twitter poll" option (by the author MB). This author was chosen given that that he has more than 2,500 followers, with the majority being gastroenterology fellows or gastroenterologists from across the world. The initial tweet described the framework of the survey in the context of the current pandemic. The questions were posted under the comments section of the initial tweet. Numerous gastroenterologists and gastroenterology fellows from across the world with prominent presence on Twitter were tagged to help disseminate the survey. Four additional questions were added based on request from other gastroenterologists. The questions were open for polling to Twitter audience for 48 hours, after which Twitter automatically closes the survey to polling. The survey was completely anonymous. The results were analyzed using the "Tweet Activity" function on Twitter.

\section{Definitions}

Regarding Twitter analytics, two definitions are important to understand. Impressions are the number of times a tweet appears on the timeline or "feed" of Twitter users. Engagements refers to the number of times a user becomes involved in a tweet. These engagements include retweets, likes, replies, or as is important to this study, poll answers.

For the purpose of this manuscript, endoscopies that were not classified urgent/emergent, or elective were described as "semi-urgent." Semi-urgent endoscopy was defined as a procedure that could reasonably be deferred for at least 8 weeks without negatively-impacting an important patient outcome (e. g. upstaging of a new cancer diagnosis).

\section{Results}

The initial Tweet had 38,795 impressions and a total of 2,855 engagements. The details of the tweet were expanded 2,291 times. The number of votes received on the initial polls ranged from 338 to 834 , providing an estimate response rate ranging from $11.8 \%$ to $29.2 \%$. The four additional questions added later had a lower response rate as expected with an average of 44 votes polled. The summary of the results is outlined in - Table 1. The actual Twitter analytics report can be accessed at: https://twitter.com/BilalMohammadMD.

Scenario 1 focused on patients with positive fecal immunohistochemistry testing (FIT) or fecal FIT-DNA test, and $70 \%$ of respondents suggested that colonoscopy was semi-urgent. Scenario 2 focused involved Barrett's esophagus with dysplasia and/or nodularity needing endoscopic treatment, and $50.4 \%$ of respondents deemed this as semi-urgent. Scenario 3 included patients with a benign ampullary adenoma needing endoscopic resection, and $53.5 \%$ of respondents voted this as semi-urgent in the current setting. Scenario 4 questioned respondents regarding patients with melena, and $44.1 \%$ thought that "any melena" needs urgent upper endoscopy, while $53.8 \%$ thought only patients with "ongoing melena" or hemodynamic instability" should undergo endoscopic evaluation. Scenario 5 discussed patients presenting with hematochezia, and the majority $(62 \%)$ thought only patients with hemodynamic instability should get inpatient colonoscopy. In patients with cirrhosis who had symptoms of upper gastrointestinal bleeding (scenario 6), 87.6\% thought that they should get urgent upper endoscopy. For patients presenting with dysphagia (scenario 7), 
- Table 1 Results of survey regarding timing of endoscopic procedures during COVID-19 pandemic.

\begin{tabular}{|c|c|c|}
\hline Indication & Most common decision & $\begin{array}{l}\text { Percentage of high- } \\
\text { est votes, } \%(n)\end{array}$ \\
\hline \multicolumn{3}{|l|}{ Gastrointestinal bleeding } \\
\hline - Evaluation of melena & Everyone should have endoscopy & $44.5(438)$ \\
\hline - Evaluation of hematochezia & Scope patients with hemodynamic instability & $61.9(389)$ \\
\hline - Evaluation of upper gastrointestinal bleeding in cirrhosis & Everyone should have EGD & $87.6(410)$ \\
\hline - Procedures for common gastrointestinal signs and symptoms & EGD for acute onset of dysphagia & $55.8(407)$ \\
\hline \multicolumn{3}{|l|}{ Evaluation of dysphagia } \\
\hline - Evaluation of iron deficiency anemia & Defer endoscopic evaluation for now & $58.3(48)$ \\
\hline - Evaluation of abnormal weight loss & Defer endoscopic evaluation for now & $84.7(359)$ \\
\hline \multicolumn{3}{|l|}{ Concerns for dysplasia/cancer } \\
\hline - Colonoscopy for positive FIT/Fecal-FIT DNA (Cologuard) testing & Defer procedure for now & $70(834)$ \\
\hline - Ulcerative colitis with dysplasia & Defer chromoendoscopy for now & $52.6(19)$ \\
\hline - EMR for colon polyps & Defer colonoscopy for now & $63.3(355)$ \\
\hline - ESD of early gastric cancer & Defer EGD for now & $58.5(340)$ \\
\hline - Endoscopic therapy of dysplastic Barrett's esophagus & Defer EGD for now & $50.4(421)$ \\
\hline - Evaluation of double duct sign with EUS & Defer EUS for now & $53.5(368)$ \\
\hline - Evaluation of ampullary adenoma & Defer endoscopic resection for now & $53.5(383)$ \\
\hline \multicolumn{3}{|l|}{ Benign [pancreatobiliary indications] } \\
\hline - Evaluation of choledocholithiasis & ERCP only if abdominal pain/jaundice & $35.7(338)$ \\
\hline - Elective exchange of biliary stenting & Defer ERCP for now & $44.1(59)$ \\
\hline - Elective removal of pancreatic stent & Defer EGD/ERCP for now & $66(50)$ \\
\hline
\end{tabular}

$55.8 \%$ of participants suggested performing esophagogastroduodenoscopy (EGD) during this time only if dysphagia was acute in onset. Scenario 8 was regarding patients with a double duct sign on cross-sectional imaging, but without a discrete mass seen, and $53.5 \%$ suggested it was semi-urgent. In patients presenting with isolated, unexplained weight loss (scenario 9), $84.7 \%$ thought this was semi-urgent. $63.5 \%$ thought that all colonic endoscopic mucosal resections (EMRs- scenario 10) could be deferred at this time. In regard to endoscopic submucosal dissection (ESD) for early gastric cancer (scenario 11), $58.5 \%$ of respondents thought this was semi-urgent. For patients with common bile duct (CBD) stones without cholangitis (scenario 12), 35.8\% thought that urgent endoscopic retrograde cholangiopancreatography (ERCP) is only needed if patients have symptoms or jaundice, $29 \%$ felt that ERCP was not urgent if no cholangitis, while $25.1 \%$ deemed all CBD stones as urgent. Scenario 13 discussed planned endoscopic removal or exchange of plastic biliary stents previously placed for scenarios currently resolved or for which the patient was currently asymptomatic. $45.6 \%$ thought this was semi-urgent and can be deferred ( $\vee$ Fig. 1 ). For patients who had a pancreatic duct (PD) stent placed during a prior ERCP (scenario 14 ), $66 \%$ re- spondents favored deferring this as semi-urgent. In patients with iron deficiency anemia (scenario 15) without overt gastroinestinal bleeding, $58.3 \%$ thought this was semi-urgent. In a patient with long-standing ulcerative colitis with recent diagnosis of dysplasia (scenario 16), $57 \%$ of respondents voted to defer performing chromoendoscopy at this time.

\section{Discussion}

Our results show that there is significant variability among gastroenterologists in regard to the timing of endoscopic procedures for semi-urgent indications during the COVID-19 pandemic. There were only three of 16 scenarios in which greater than $70 \%$ of gastroenterologists agreed on procedure timing. These scenarios were deferring colonoscopy for patients who had fecal FIT-DNA or FIT positive testing, performing urgent endoscopy for patients with cirrhosis presenting with melena and hematemesis, and deferring endoscopic evaluation for unexplained isolated weight loss. In regard to other scenarios such as the endoscopic treatment for patients with dysplastic Barrett's esophagus, patients with ampullary adenoma needing resection and patients with double duct sign on imaging (without 


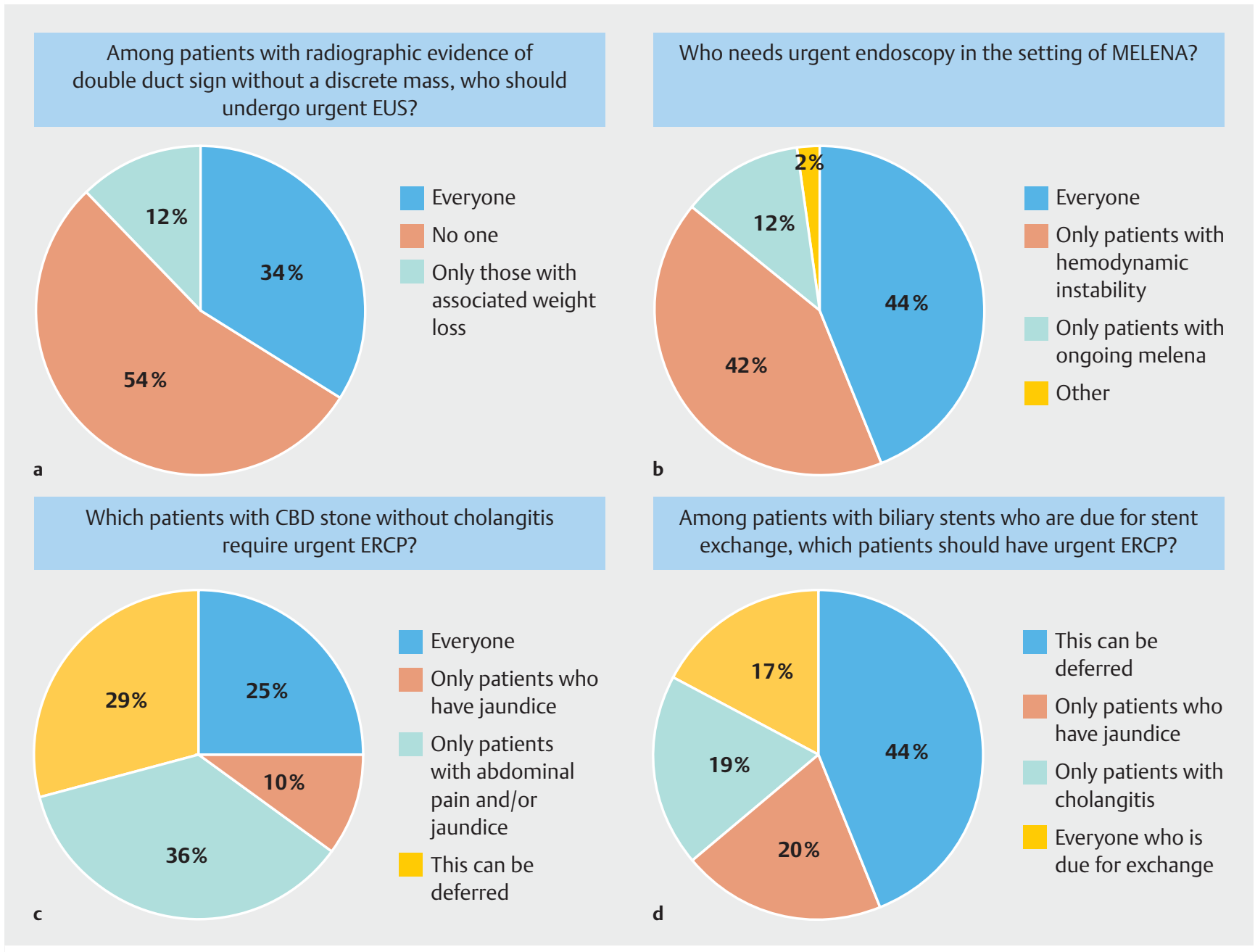

Fig. 1 Demonstration of variation in gastroenterologists regarding timing of procedures for semi-urgent procedural indications.

a focal mass), approximately $50 \%$ to $55 \%$ of the gastroenterologists thought these procedures were semi-urgent and should be deferred. While approximately half of respondents thought that procedures for these indications should be performed in select situations during this time.

Gastrointestinal bleeding is routinely considered an indication for urgent endoscopy. However, in our survey regarding patients presenting with hematochezia, the majority of respondents indicated that colonoscopy should only be pursued if a patient has hemodynamic instability. Similarly, in patients with melena, fewer than half (44\%) of respondents thought that every patient with melena warranted endoscopy, while the remainder opted for endoscopy only if ongoing signs of melena or hemodynamic instability were present. These findings are interesting since they might represent a change in typical gastroenterology management pathways necessitated by the COVID19 pandemic.

The highest degree of variability was seen in answers related to ERCP in patients with CBD stone without cholangitis. Onefourth of respondents indicated that all CBD stones are urgent, while $45 \%$ suggested performing ERCP only if symptoms were present, and approximately $30 \%$ indicated that ERCP was semi-urgent if cholangitis was not present. This highlights that there is currently no consensus on optimal timing for ERCP in patients with asymptomatic choledocholithiasis, even prior to the COVID-19 pandemic.

Another interesting finding was that ESD for early gastric cancer was suggested to be deferred by $58 \%$ of respondents at this time, but $35 \%$ of respondents indicated this was an urgent procedure. One hypothesis for this could be that ESD can be a long procedure and it might concern gastroenterologists that longer procedure times might increase risk of COVID-19 transmission. Secondly, ESD also carries a higher AE event rate than routine endoscopy [10], so there could be concerns that in case of complications such as perforation, there would be utilization of the operating room services in an already resource-constrained environment. It is interesting to note that the highest degree of variation in responses was for premalignant conditions such as Barrett's esophagus, ampullary adenomas, and ulcerative colitis with dysplasia. This suggests that there is some uncertainty among gastroenterologists regarding deferring treatment of premalignant conditions in the current environment. 
There was also variation in answers in regard to patients who underwent prior ERCP with biliary and PD stent placement. While most respondents suggested these procedures should to deferred during this time, some indicate that ERCP for biliary stent exchange and PD stent removal should be prioritized and performed during this period.

There could be several reasons for variations in responses seen in our survey. The respondents are from all over the world and there may be practice variation in different regions of the world. In addition, the COVID-19 pandemic is in different phases throughout the world and as the crisis worsens, the definition of semi-urgent endoscopy may narrow. It's plausible that respondents from hard-hit Western European countries such as Spain and Italy have a stricter definition of what warrants endoscopic evaluation during this pandemic. This cross-sectional analysis captures the opinion of the respondents at a specific time in the pandemic, the severity of which varies by locality. As the crisis worsens, a longitudinal study may well show that these opinions on what constitutes "semi-urgent" endoscopy narrow over time as disease prevalence increases. The variability of responses could also be driven by the fact that currently there is no defined duration for this pandemic and some gastroenterologists might be concerned about the uncertainty of how long a deferred patient would have to wait until their procedure is finally performed. This becomes especially important in patients with premalignant conditions. The Joint GI Society Message on COVID-19 does state that some non-urgent procedures are higher priority and examples included prosthesis (e.g. stent) removal and evaluation of significant symptoms [11]. However, as the number of COVID-19 cases are exponentially rising in many regions, another major concern for performing any endoscopy is the amount of personal protective equipment (PPE) that is needed to perform a single procedure safely. PPE is an important resource at this time which needs to be judiciously used. Hence, many gastroenterologists are opting to defer many of the procedures above, even though in the "non-pandemic" situation these are likely to be performed sooner.

Our study has several limitations. Given that this survey was conducted on Twitter, we do not have an exact response rate. We did, however, use the engagements and the number of times the details of tweets were expanded on the initial Tweet as the denominator and number of votes as the respondents to estimate the response rate. Our estimated response rate is low, however, previously reported response rates are applicable to traditional methods like email and regular mail, and may not apply to social media platforms like Twitter. This highlights the need to develop new standards of data acquisition/surveys as social media platforms are going to be increasingly and effectively used for this purpose in the future. Also, we cannot tell how many of the people who cast votes were from which countries and what level in their career (gastroenterologists, gastroenterology fellows, internal medicine physicians). Expanding on this, there were poll questions regarding procedures such as ERCP and ESD, which a minority of the respondents actually perform in clinical practice. It is unclear whether answers would vary if only physicians credentialed in these techniques were al- lowed to respond. As previously mentioned, this crisis is in different phases throughout the world. At the time of this manuscript, Spain and Italy continue to have dire situations regarding PPE availability, and inpatient volume has exceeded the critical care threshold capacity in many of these centers. We were not able to stratify responses by geographical region to assess for variability, which would have certainly provided important information. In addition, releasing a poll on Twitter will only capture physicians who are currently engaged in using this particular social media platform, and the popularity of Twitter use varies among different countries. However, we did find significant engagement from across the world (North America, South America, Europe, Asia, Africa and Australia), and participation from gastroenterologists from both the academic and community setting.

\section{Conclusion}

Despite these limitations, our analysis provides a real-time snapshot of the current thoughts of gastroenterologists around the world during the COVID-19 pandemic. It also highlights that there is currently a lack of consensus regarding how to prioritize certain potentially time-sensitive endoscopic procedures. Although each patient is unique and many clinical decisions must be made on a case-by-case basis, our analysis will provide some perspective and guidance to gastroenterologists while dealing with these clinical scenarios. Our findings also strongly support the need for developing societal guidance in these "semi-urgent" scenarios to assist during the current COVID-19 pandemic, and we are aware of numerous gastroenterology societies that are engaged presently in this endeavor. Finally, this study shows how social media platforms can be positively used to gain instantaneous and clinically useful information from around the globe in response to rapidly changing situations.

\section{Acknowlegements}

The authors thank Dr Heiko Pohl for his critical review of manuscript and feedback in improving the manuscript.

The authors also acknowledge the gastroenterologists and GI fellows from around the world who participated in this survey.

\section{Competing interests}

The authors have no relevant conflicts of interest to this manuscript. But for details, author TB is consultant for Boston Scientific and Medtronic. Author DP is Disclosures of Conflict of Interest is a consultant for Boston Scientific, Olympus, Fuji Film, Nine Point, CSA, Medtronic. The remaining authors have no conflicts of interest.

\section{References}

[1] World Health Organization. Coronavirus disease (COVID-2019) situation report - 63. Available at (Accessed March 24, 2020): https:// www.who.int/docs/default-source/coronaviruse/situation-reports/ 20200323-sitrep-63-covid-19.pdf?sfvrsn=b617302d_2 
[2] Li R, Pei S, Chen B et al. Substantial undocumented infection facilitates the rapid dissemination of novel coronavirus (SARS-CoV2). Science 2020: doi:10.1126/science.abb3221 [Epub ahead of print]

[3] Wang D, Hu B, Hu C et al. Clinical characteristics of 138 hospitalized patients with 2019 novel coronavirus-infected pneumonia in Wuhan, China. JAMA 2020: doi:10.1001/jama.2020.1585 [Epub ahead of print]

[4] Soetikno R, Teoh AY, Kaltenbach T et al. Considerations in performing endoscopy during the COVID19 pandemic. Gastrointestinal Endoscopy 2020: doi:10.1016/j.gie.2020.03.3758 [Epub ahead of print]

[5] Gu J, Han B, Wang J. COVID-19: Gastrointestinal manifestations and potential fecal-oral transmission. Gastroenterology 2020: doi:10.1053/j.gastro.2020.02.054 [Epub ahead of print]

[6] Repici A, Maselli R, Colombo M et al. Coronavirus (COVID-19) outbreak: what the department of endoscopy should know. Gastrointest Endosc 2020: doi:10.1016/j.gie.2020.03.019 [Epub ahead of print]
[7] Adler DG, Leighton JA, Davila RE et al. ASGE guideline: The role of endoscopy in acute non-variceal upper-Gl hemorrhage. Gastrointest Endosc 2004; 60: 497-504

[8] Stanley A], Laine L. Management of acute upper gastrointestinal bleeding. BMJ 2019; 364: 1536

[9] Iqbal U, Khara HS, Hu Y et al. Emergent versus urgent ERCP in acute cholangitis: a systematic review and meta-analysis. Gastrointest Endosc 2020; 91: 753-760 e4

[10] Draganov PV, Wang AY, Othman MO et al. AGA Institute Clinical Practice Update: Endoscopic Submucosal Dissection in the United States. Clin Gastroenterol Hepatol 2019; 17: 16-25.e1

[11] Joint GI Society. Message on COVID-19. Available at (Accessed March 24, 2020): https://gi.org/2020/03/15/joint-gi-society-message-oncovid-19/ 